\title{
International Space Station U.S. GN\&C Momentum Manager Controller Design for Shuttle Thermal Protection System Repair
}

\author{
Christopher R. Sims ${ }^{*}$ \\ The Boeing Company, Houston, TX, 77059
}

\begin{abstract}
This paper describes the design of the ISS Momentum Manager controllers for the Orbiter Repair Maneuver (ORM) and Orbiter Tile. Repair operations. Momentum Manager Controllers provide non-propulsive attitude control via CMGs. Non-propulsive control is required at the beginning and the middle of the ORM and at the tile repair position. This paper first reviews the design issues and requirements, then presents the design methodology, and concludes with analysis results that verify the design.
\end{abstract}

\section{Introduction}

For Space Shuttle Flight LF1, an on-orbit repair capability for the Shuttle's Thermal Protection System (TPS) was desired. The accepted solution was to perform the repair while the Shuttle is at the International Space Station (ISS). The repair would be done by an astronaut attached to the Space Station Robotic Manipulator System (SSRMS). The SSRMS does not have enough reach capability to reach potential repair sites with the Space Shuttle docked to the available docking ports. This is overcome by utilizing the Shuttle Robotic Manipulator System (SRMS). The SRMS grapples the Station while the Shuttle undocks from the ISS. The SRMS will then effectively flip the Shuttle upside down exposing the underside of the Shuttle to the reach space of the SSRMS while ISS maintains attitude control. This robotic operation is called the Orbiter Repair Maneuver (ORM). In order to make it more operationally feasible, an overnight park position happens midway through the maneuver.

The ISS should utilize its non-propulsive attitude control capability at Shuttle unberthing, Shuttle berthing, the ONP, and the final repair position. This paper describes the design and analysis of the controllers that were made to provide non-propulsive attitude control for these stages of the TPS repair.

\section{Designs}

The ISS provides non-propulsive attitude control via a linear control law called Momentum Manager. This control law is based on the design detailed in [1].

Momentum Manager Controller designs for use in the TPS repair operations have several design issues that all ISS Momentum Manager designs have as well as unique ones due to the repair operations. The following list captures general ISS momentum manager design goals:

- No steady state CMG desaturations (non-propulsive goal)

- Maintain attitude envelopes in startup and steady state

- Peak steady CMG momentum levels should allow for single CMG failure

- Minimize attitude variation and attitude rate

Design trades exist between all these mentioned goals and these trades are bounded by the vehicle angular dynamics of earth oriented orbiting spacecraft and expected aerodynamics torque loading. For a more detailed discussion see [1].

\footnotetext{
${ }^{*}$ Engineer/Scientist III, ISS GN\&C Systems Integration, Houston, TX, Not a Current AIAA Member
} 
For TPS repair operations new design challenges exist. One being the SRMS dynamics, its flexible dynamics are located near the bandwidth of the standard MM designs $(0.007 \mathrm{rad} / \mathrm{sec})$. Figure 1 shows the SRMS flex dynamics that are added compared to the berth model which has no SRMS flex dynamics. The MM design should avoid unstable or poor interaction with the SRMS. This challenge is made more difficult due to the nonlinear behavior of the SRMS joints and the set of design tool utilized assuming linear behavior. The next design challenge is related to the positioning of the Orbiter by the SRMS determines the overall mated stacks inertia tensor properties. This impacts on how well the mated vehicles attitude motion generates secular Gravity gradient torques to oppose secular aerodynamic torques which can not be rejected by $\mathrm{CMG}$ torques. This makes the design goal of keeping

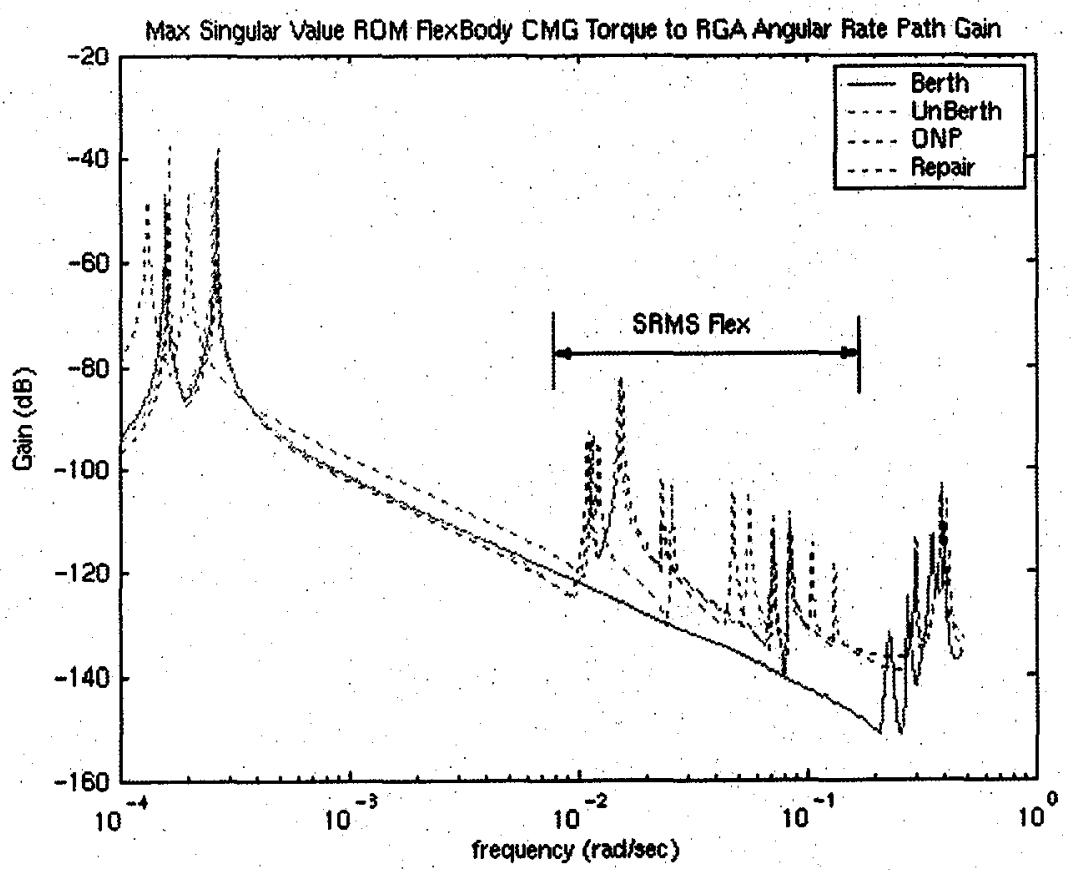

small vehicle attitude variations and angular rates a difficult challenge under expected uncertainties.

\section{A. CONTROLLER ARCHITECTURE}

The controller architecture available in the ISS U.S. GN\&C Flight software provides high level of flexibility to overcome many design issues to be encountered during the assembly of the ISS. This paper will be focusing specifically the architecture that supports non-propulsive attitude control. The actuators utilized are 4 double gimbaled CMGs. The linear controller is full state feedback controller which has access to the 9 controlled variables - attitude, attitude rate, and CMG momentum. The linear controller also utilizes the provided 3 integrators, 3 orbit frequency disturbance rejection filters, 3 twice orbit disturbance rejection filters all implemented in the LVLH frame [1]. Three $5^{\text {th }}$ Order filters are located in each of the axis of the angular vehicle rate signal for use in flex structure filtering. Also three $5^{\text {th }}$ Order filters are located in each of the axis of the Torque command coming from the linear rigid body controller for use in flex structure filtering. The rigid body gains and flex filter parameters are packaged in one uplinkable file. Thus having a unique flex filter for each set of rigid body gains does not complicate loading new MM designs into the ISS GN\&C flight computer. Figure 2 shows a simplified closed loop diagram of the control system and linear flex body plant. 


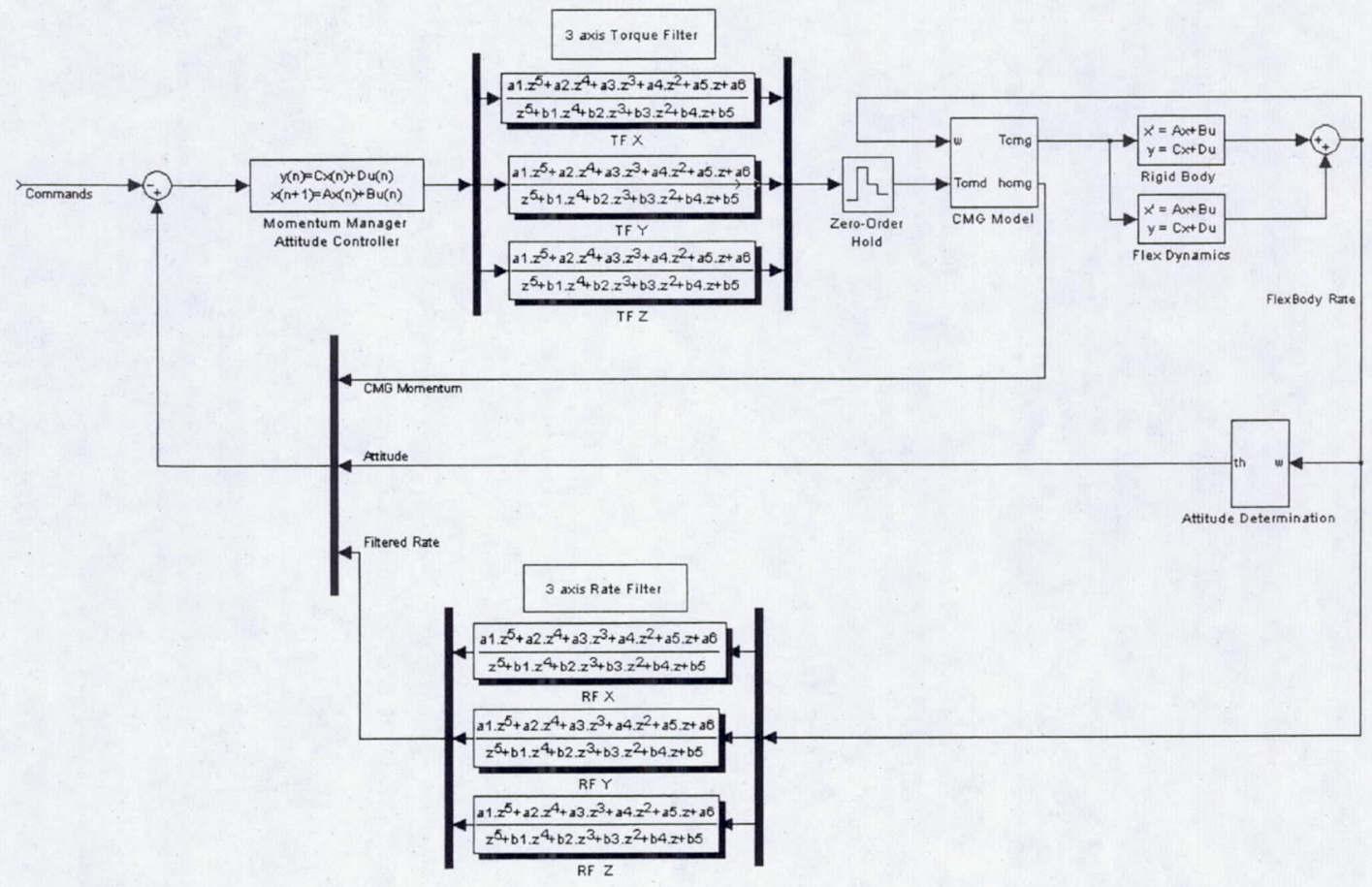

Figure 2. ISS U.S. GN\&C Momentum Manager Controller System Architecture

\section{B. MM Rigid Body Designs}

\section{BERTH/UNBERTH Positions}

This particular operation provided an unique design challenge wrt to previous designs. To minimize loading of the docking mechanisms while maintaining attitude control, it was desired that non-propulsive control during berthing and unberthing operations. During the design phase it was desired that non-propulsive control be maintained until the Shuttle is been removed $15 \mathrm{ft}$ away from its docked position. In order to provide one controller design that provides acceptable robustness at both docked and $15 \mathrm{ft}$ out convex optimization was utilized in determining the state feedback gains.

The standard objective function to minimize in LQR controller design is to solve for a static state feedback gain that minimizes

$$
J=\int_{t 0}^{\infty}(x Q x+u R u) d t
$$

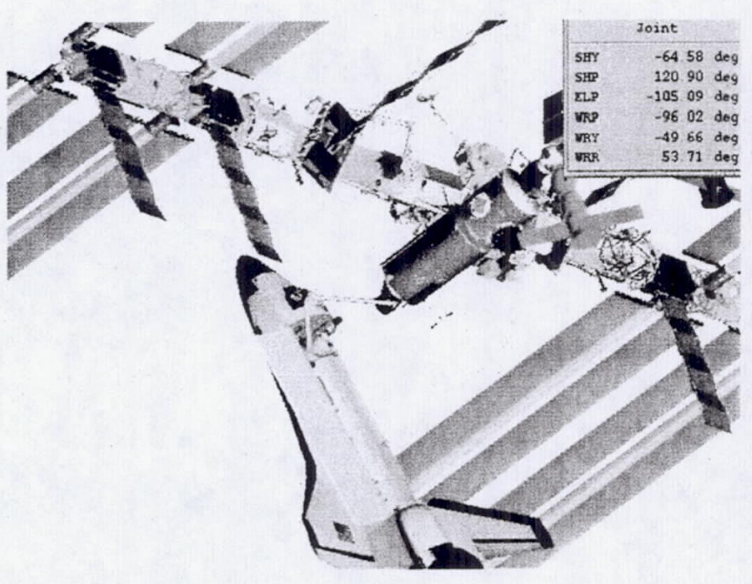

Figure 3. ISS w/ Orbiter in Unberth Position

for a given plant defined by

$\dot{x}=A x+B u$ with control law $u=K x$

For a single MM design for these two configs, we looked to find a state feedback controller $\mathrm{K}$ that will stabilize the plants while minimize the cost function for the two different systems.

Per [2] a suboptimal controller that minimizes this objective function for a polytopic plant can be solved for by the following constrained minimization problem 
Minimize a dummy variable $v$ with the decision variables $X$ and $K$ under the following constraints.

$$
\begin{aligned}
& {\left[\begin{array}{cc}
A_{\text {berth }} X+X A_{\text {berth }}{ }^{T}+B_{\text {berth }} Y+Y^{T} B_{\text {berth }}{ }^{T}+ & \left(Q^{1 / 2} X+R^{1 / 2} Y\right) \\
Q^{1 / 2} X+R^{1 / 2} Y & -I
\end{array}\right]>v I} \\
& {\left[\begin{array}{cc}
A_{\text {unberth }} X+X A_{\text {unberth }}{ }^{T}+B_{\text {unberth }} Y+Y^{T} B_{\text {unberth }}{ }^{T}+ & \left(Q^{1 / 2} X+R^{1 / 2} Y\right) \\
Q^{1 / 2} X+R^{1 / 2} Y & -I
\end{array}\right]>v I}
\end{aligned}
$$

$X>0$

$v>0$

where

$$
K=Y X^{-1}
$$

\section{ONP Position}

The rigid body controller designs for the overnight park were based off of disturbance rejection design principles as discussed in [2] and utilized LQR solver for synthesis of state feedback gains. Three designs were made available:

a. A Momentum emphasis design which emphasized maintaining low $\mathrm{CMG}$ momentum.

b. A Power Generation design which emphasized biased roll attitude to orient the solar arrays to towards the sun for positive solar beta angles

c. A Power Emphasis design which biased the roll attitude to orient the solar arrays toward the sun for negative solar beta angle conditions.

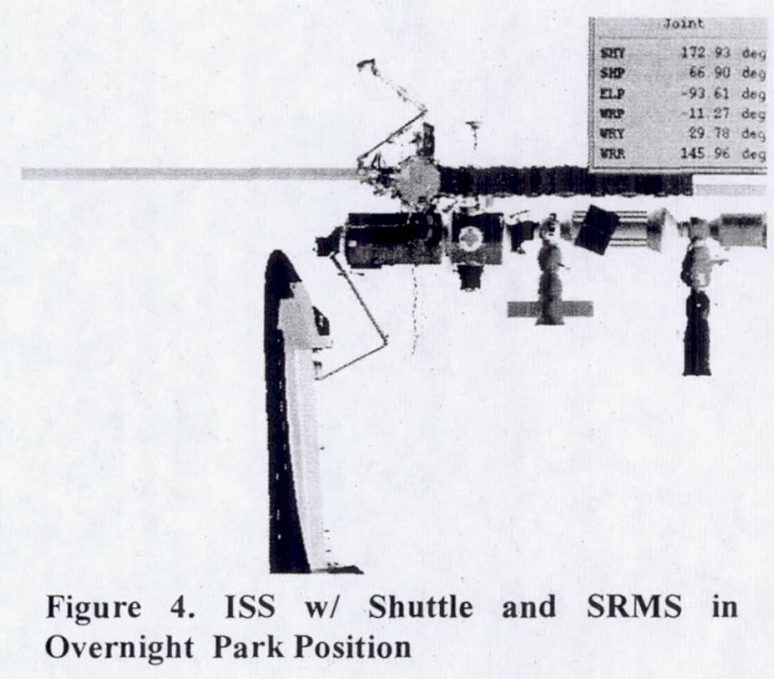

Orbiter Repair Position

The rigid body controller designs for the Orbiter repair positions were based off of disturbance rejection design principles as discussed in [2] and utilized LQR solver for synthesis of state feedback gains. Three designs were made available

- A Power Generation design which emphasized biased roll attitude to orient the solar arrays to towards the sun for positive solar beta angles

- A Power Emphasis design which biased the roll attitude to orient the solar arrays toward the sun for negative solar beta angle conditions.

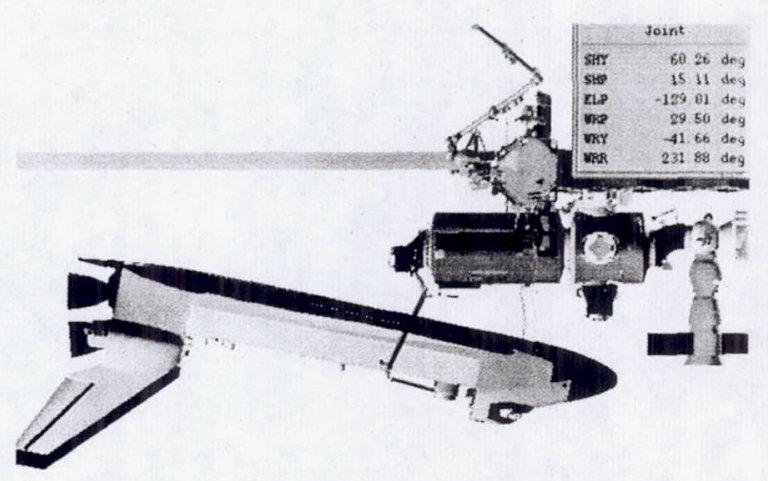

Figure 5. ISS w/ Shuttle and SRMS in Orbiter Repair Position 


\section{FLEX FILTER DESIGNS}

Some iterations of the rigid body controller designs were made to minimize interaction with the low frequency SRMS arm dynamics, yet the flex filter designs provided the main solution to avoid controller structure interaction. Due to the uncertainty of the linearized SRMS flex dynamics all three axis utilize the same filter design. Fixed structure and fixed order filter synthesis has not been solved generally. Therefore flex filter designs were done by manual iterations of flex filters poles and zeros to achieve desired single loop torque channel cut stability margins of $6 \mathrm{db}$ and $30^{\circ}$ of phase margin. The linear flex dynamics being very well coupled to the CMG actuator, and the limited order of the flex filters does not provide enough attenuation to gain stabilize these dynamics. The dominant SRMS flex modes were gainphase stabilized by the flex filters.

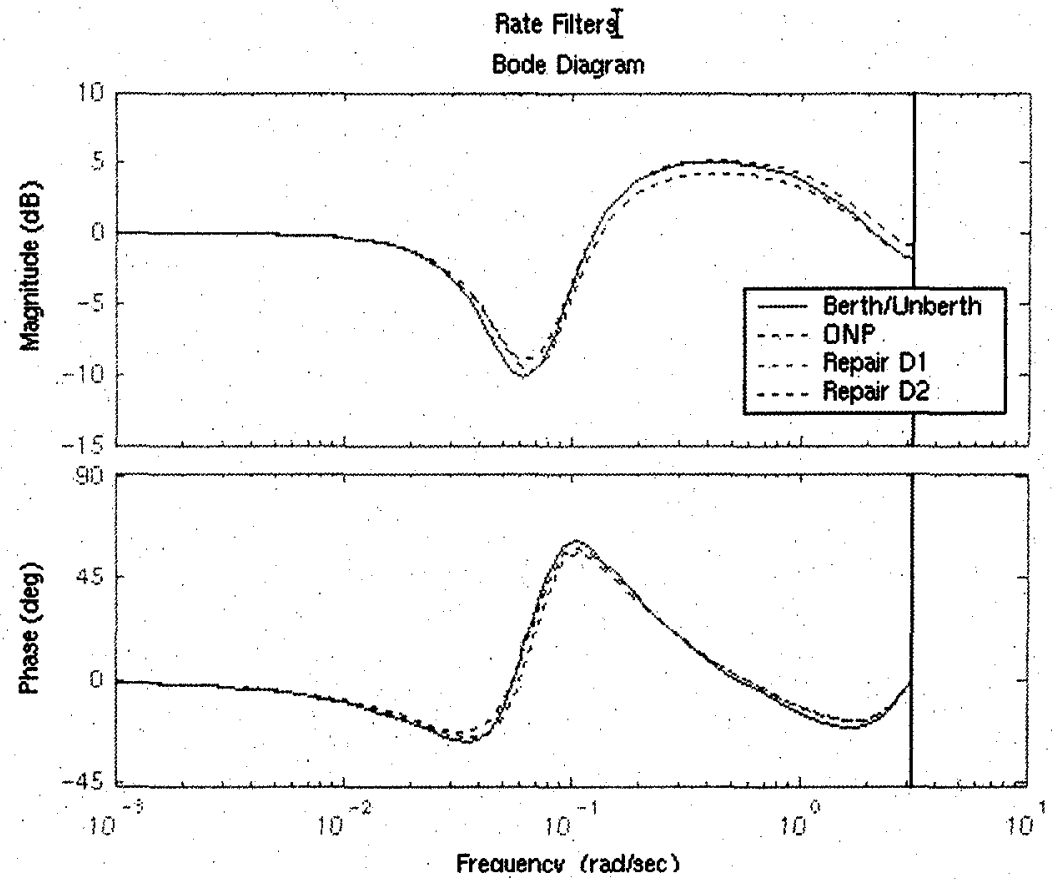

Figure 6. Rate Filter Designs for MM Bode Diagrams

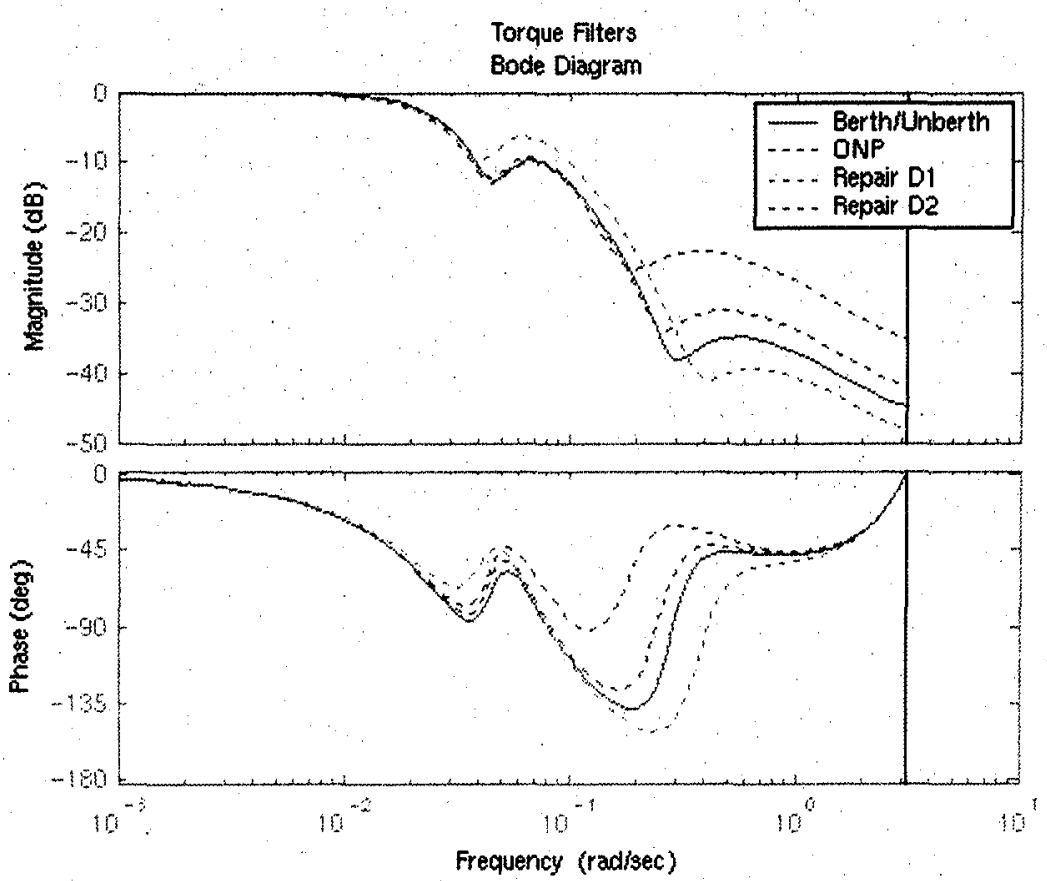

Figure 7. Torque Filter Designs for MM Bode Diagrams 


\section{Analysis}

\section{A. FLEX BODY ROBUST STABILITY}

Linear stability analysis was performed to ensure the closed loop system has reasonable margins to plant and modeling uncertainties. Multi-loop cut gain and phase margins based off characteristic loci methods were taken for nominal rigid body plant with both a nominal flex structure model and perturbed model. Both the perturbed and nominal flex structure models utilized $0.5 \%$ damping coefficient. The perturbed flex models assumed $+50 \%$ modal amplitude and $+1-20 \%$ modal frequency. The flexbody were taken in the 3 axis torque channel Nyquist cut.

The minimum absolute value of characteristic loci gain margins were above $6 \mathrm{~dB}$. The lowest characteristic loci phase margins were just below 30 degrees at 29.4 degrees. Below are a few sample characteristic loci plots with their corresponding margin takeoffs noted.

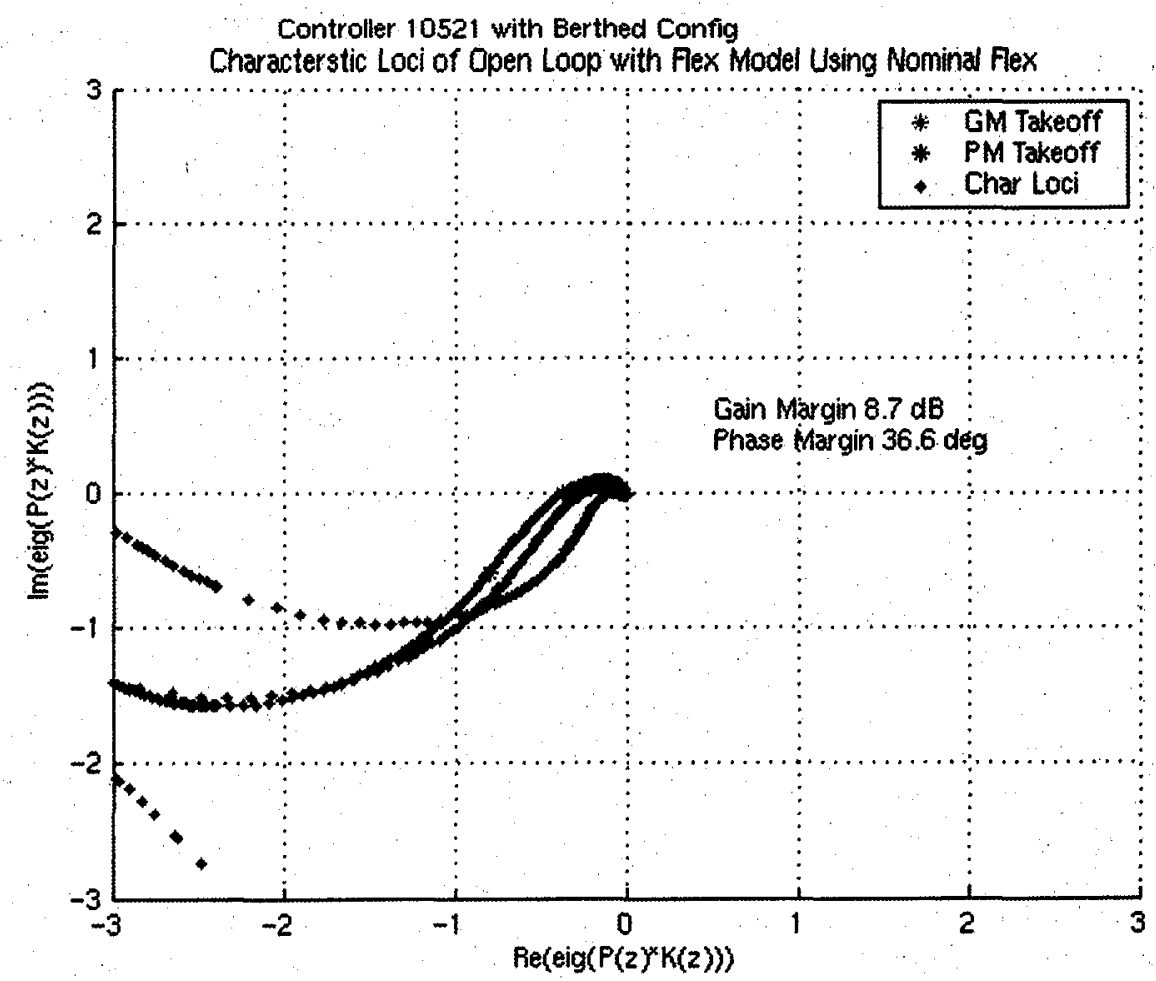



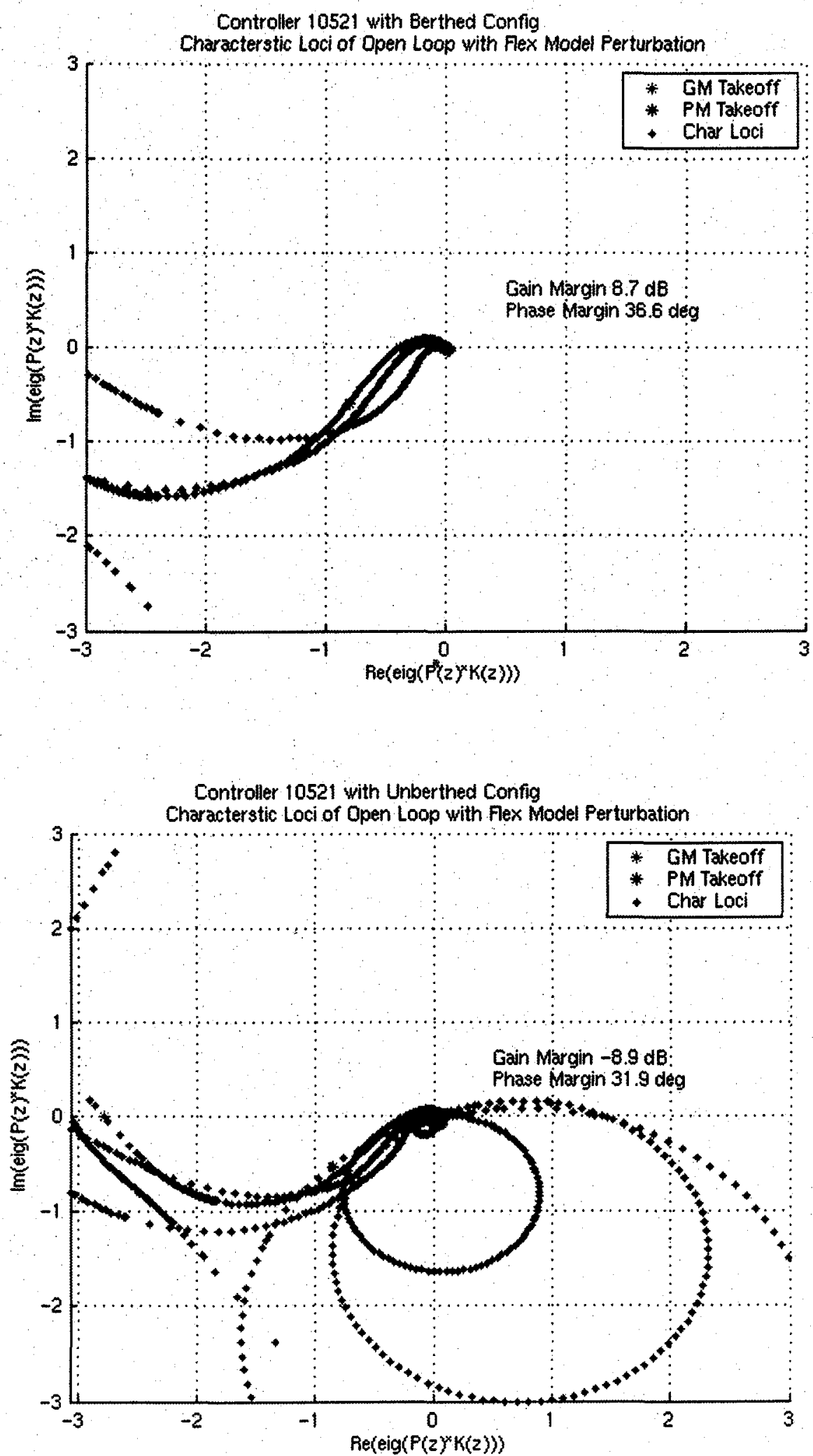


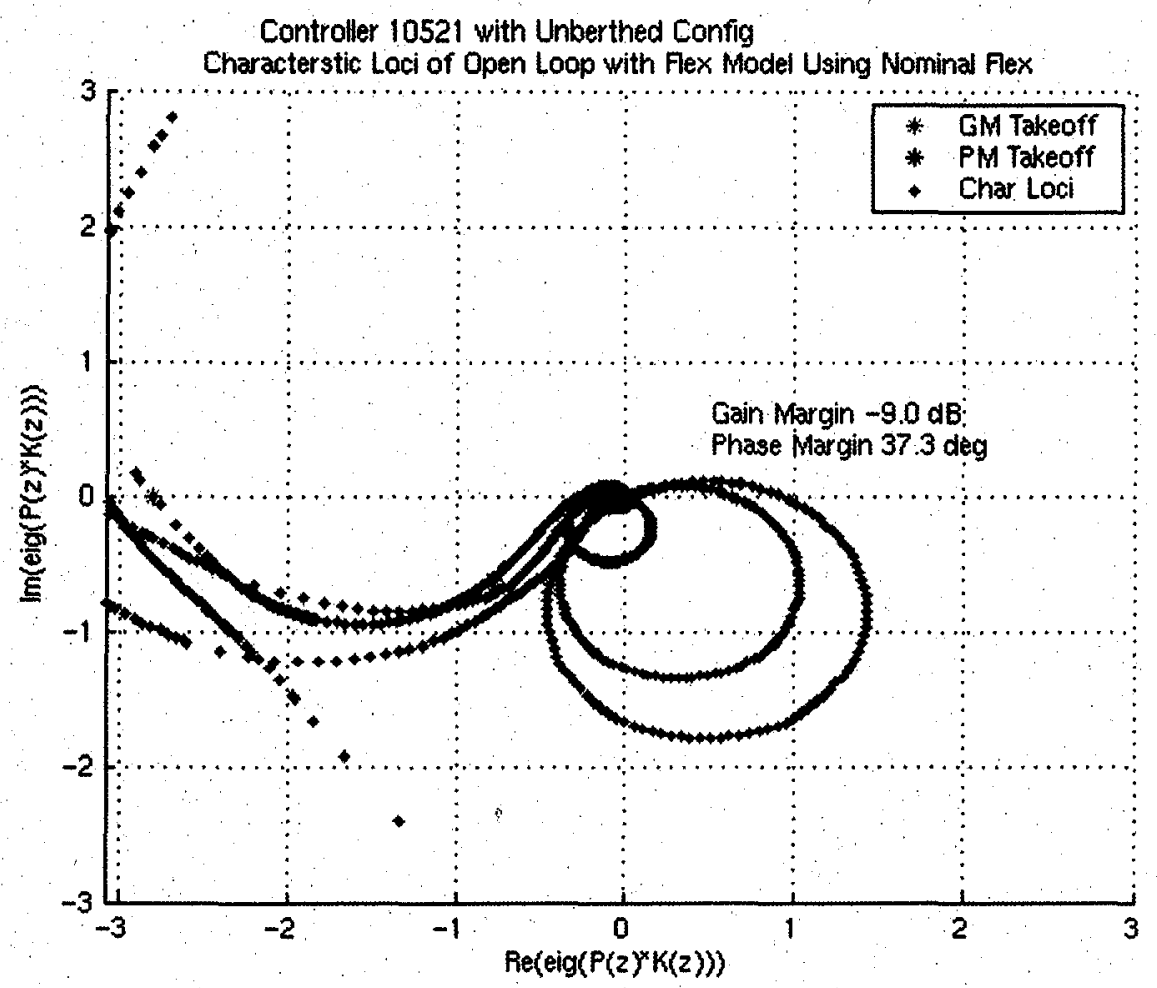

Controller 10522 with ONP Config

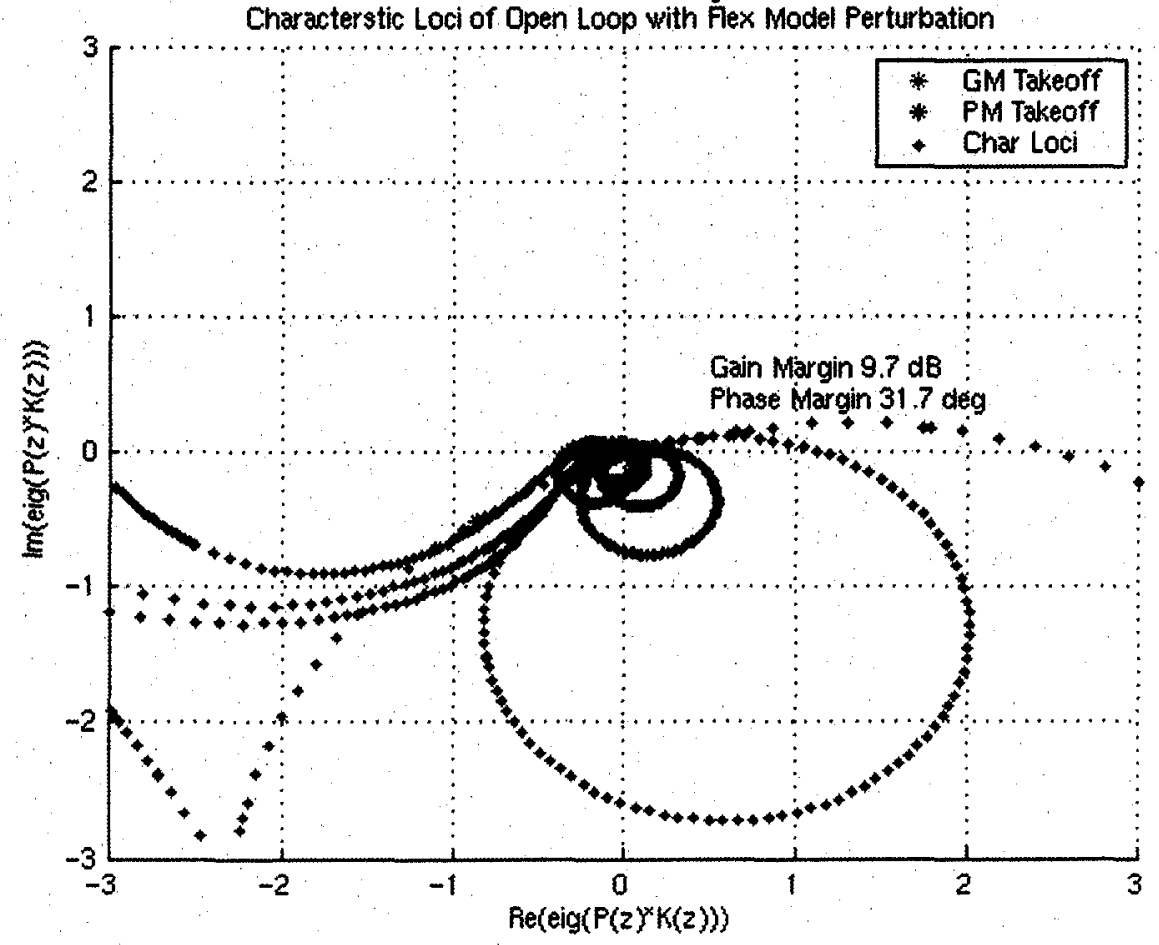




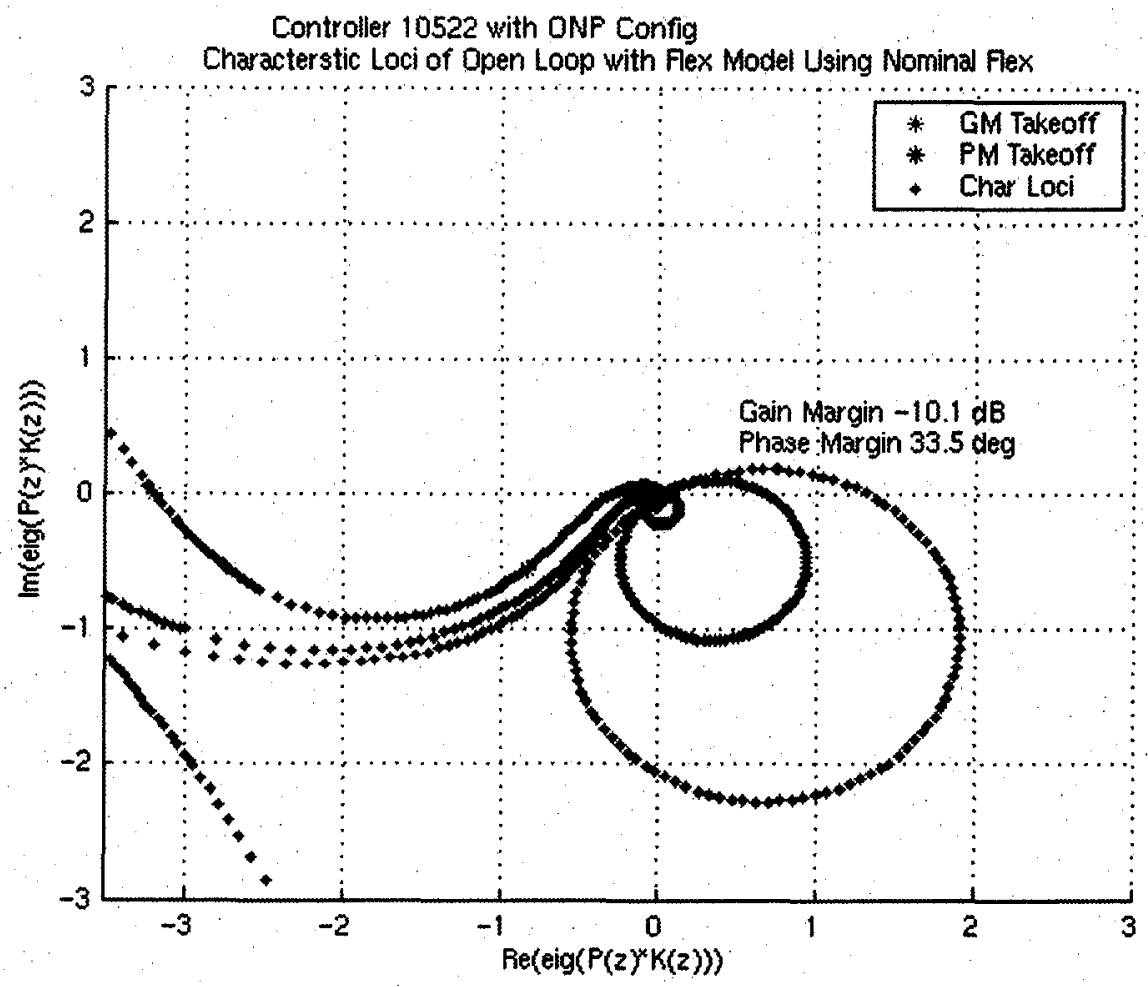

Controller 10525 with Repair Config

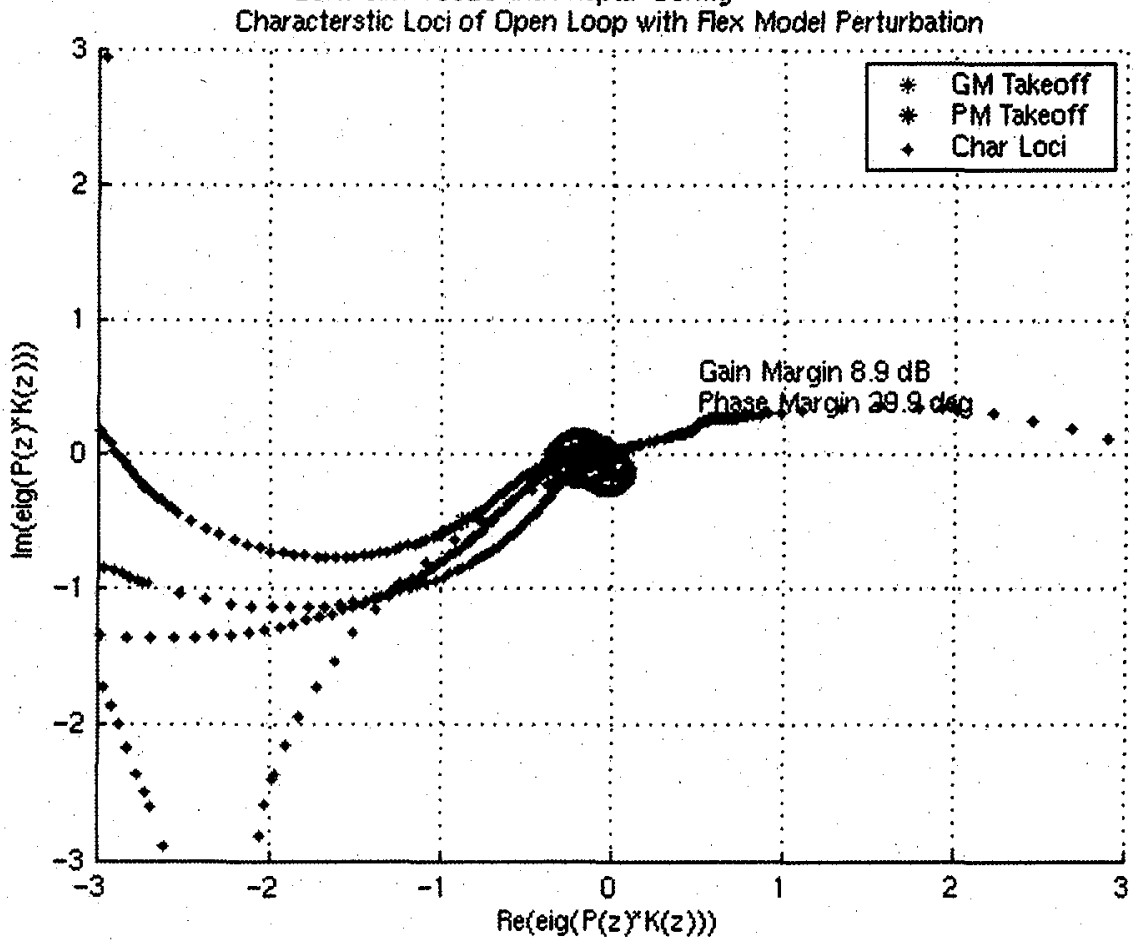




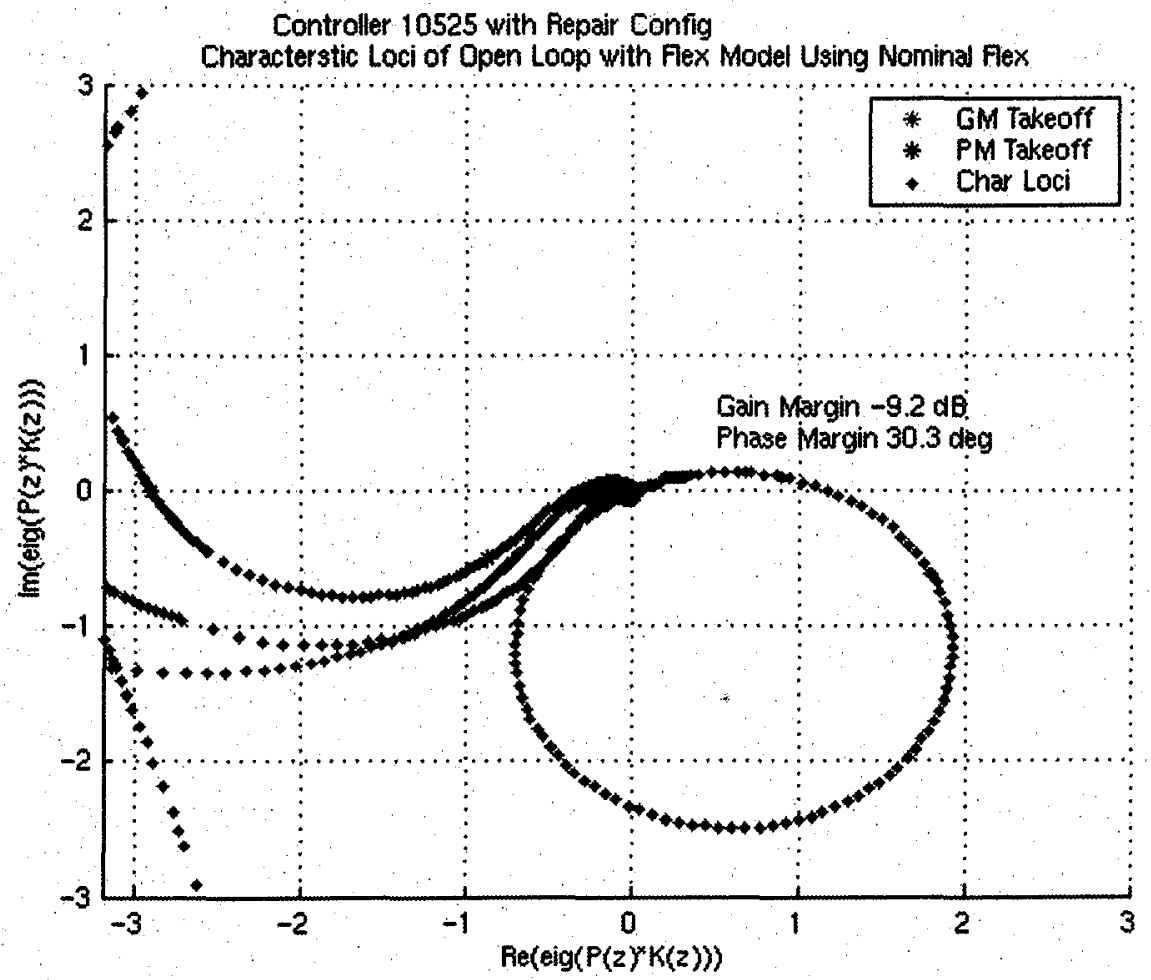

Also since the gain margins were taken at the conservative extremes of the flex structure model uncertainties, in order to ensure that some combination of perturbations within the perturbation ranges doesn't cause instability. A continuous LFT model was constructed that perturbed the modes above the Nyquist sample frequency. $\mu$-analysis was performed with this real value structured uncertainty. The results from this analysis indicate that the uncertainty range for the flex mode uncertainties of modal amplitude of $+/-50 \%$ and $+/-20 \%$ of modal frequency could nearly be doubled and still maintain stability (performance is not guaranteed though):

\section{B. RIGID BODY ROBUST PERFORMANCE}

\section{LINEAR ANALYSIS}

To ensure the MM controller's performance to known parametric uncertainties a steady state linear performance analysis was performed. Orbit rate harmonic composition of external disturbance torques is calculated and input into a linear model. The resultant output is the expected steady state performance of the closed loop system.. This linear model perturbed through 192 permutations of parameter perturbations of composite vehicle Moments and Products of inertia, atmospheric density multipliers, and a ratio of specular to diffuse atmosphere interaction with ISS structure. The result of the 192 cases for each controller design under 3 different atmospheric conditions max, min, and nom show all steady state guidelines and goals mentioned above were met:

\section{TIME DOMAIN NON-LINEAR ANALYSIS}

Also since these controllers were designed not interact with the passively stable flex structure, rigid body dynamics would dominate especially at steady state. Thus rigid-body multi-degree of freedom non-linear simulation analysis were still of value to ensure robustness to atmospheric conditions with higher fidelity nonlinear models. Results from these simulations show acceptable performance.

\section{FLEX BODY PERFORMANCE}

To verify the designs a high-fidelity non-linear flex body simulation was utilized to capture a high level of accuracy. NASA JSC Engineering Directorate owns and maintains and simulation called SOMBAT. This model capture multi-body coupled non-linear flex body dynamics of the ISS and its articulating bodies. It contains a complete model of the ISS U.S. GN\&C Control Attitude FSW. It has also has integrated into it high fidelity flex body models of the SRMS and SSRMS dynamics and their corresponding flight software. The high-fidelity robotic 
arm models contain the non-linear joint stiffness dynamics which will dominate the flexible relative motion between ISS and Shuttle.
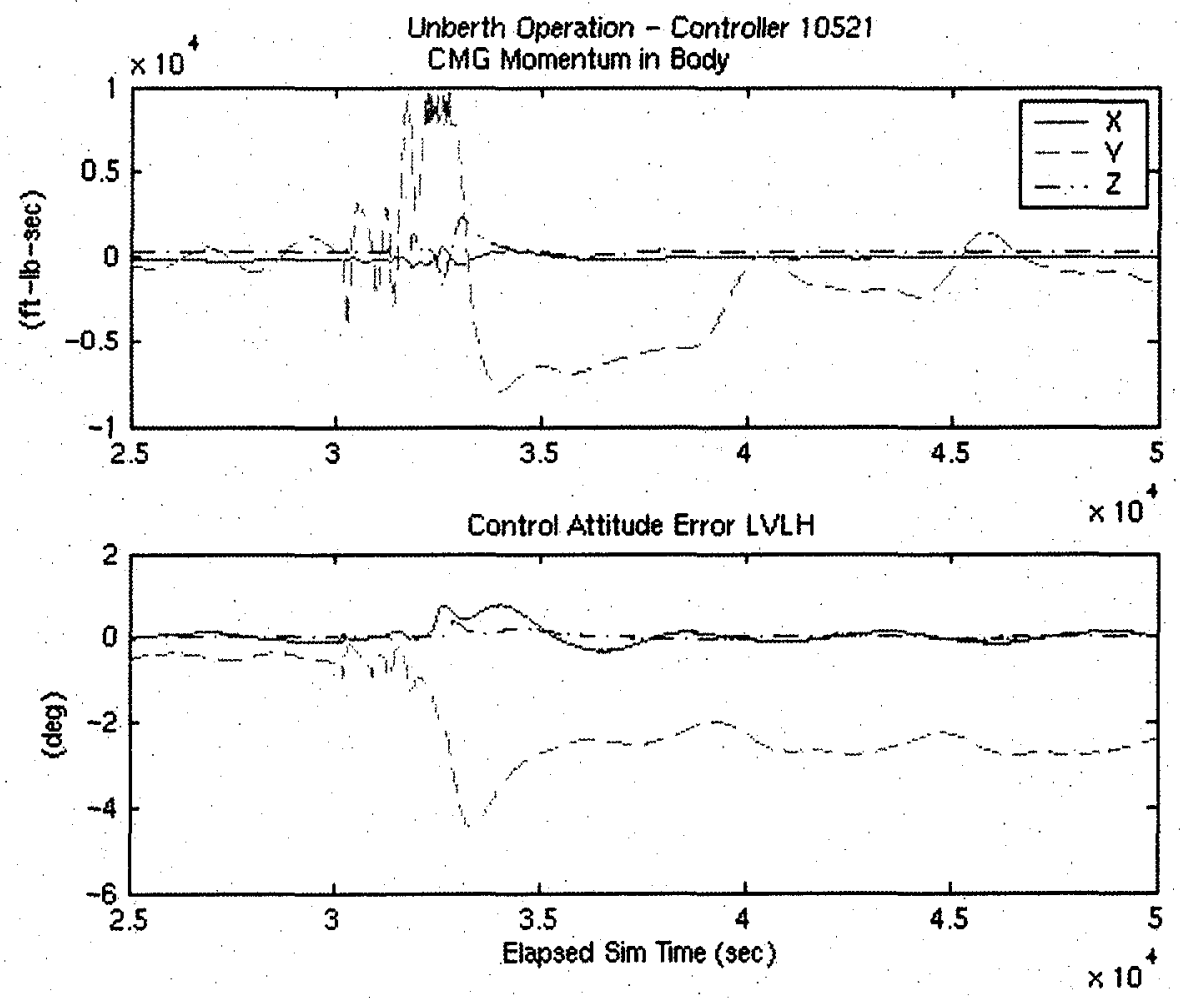

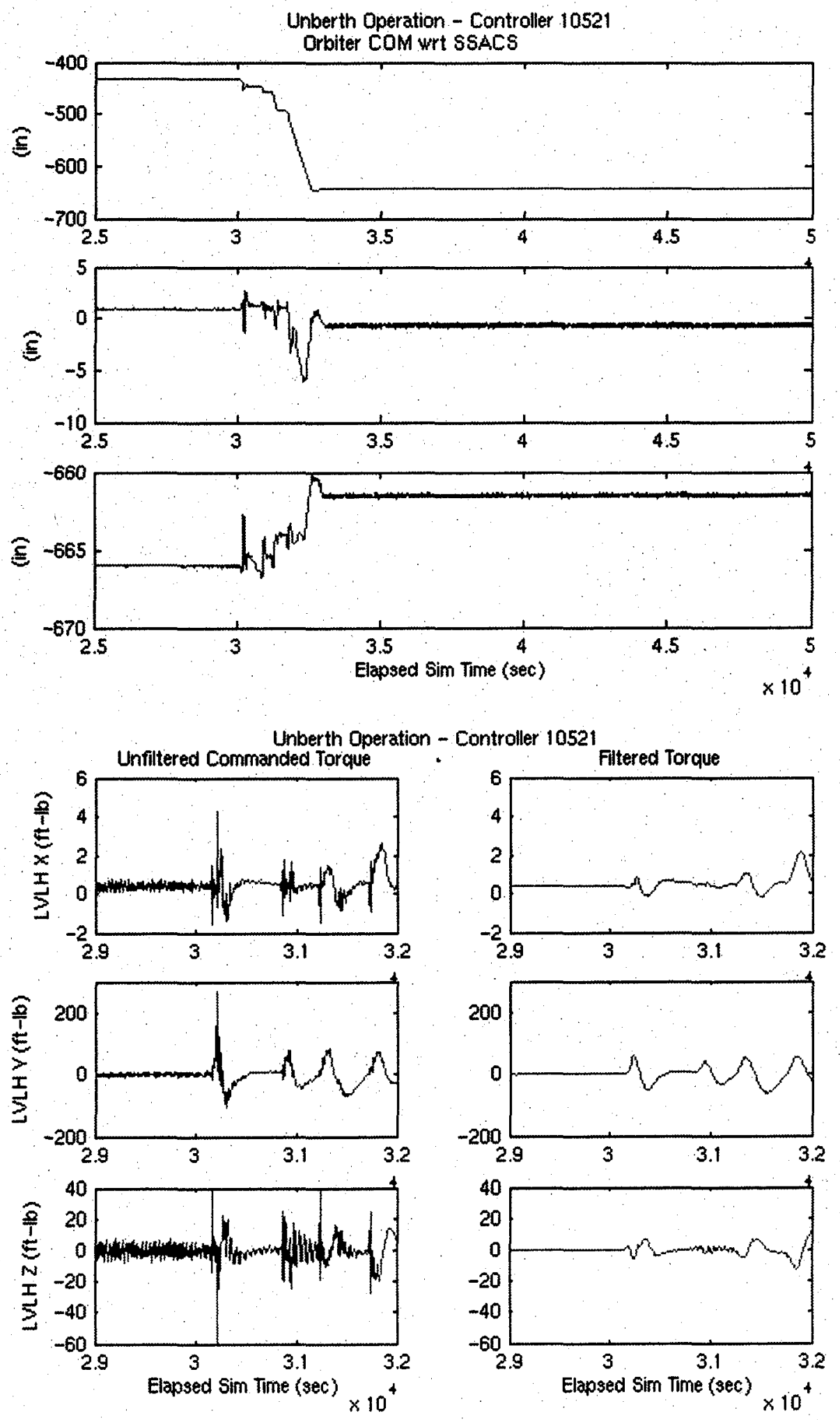

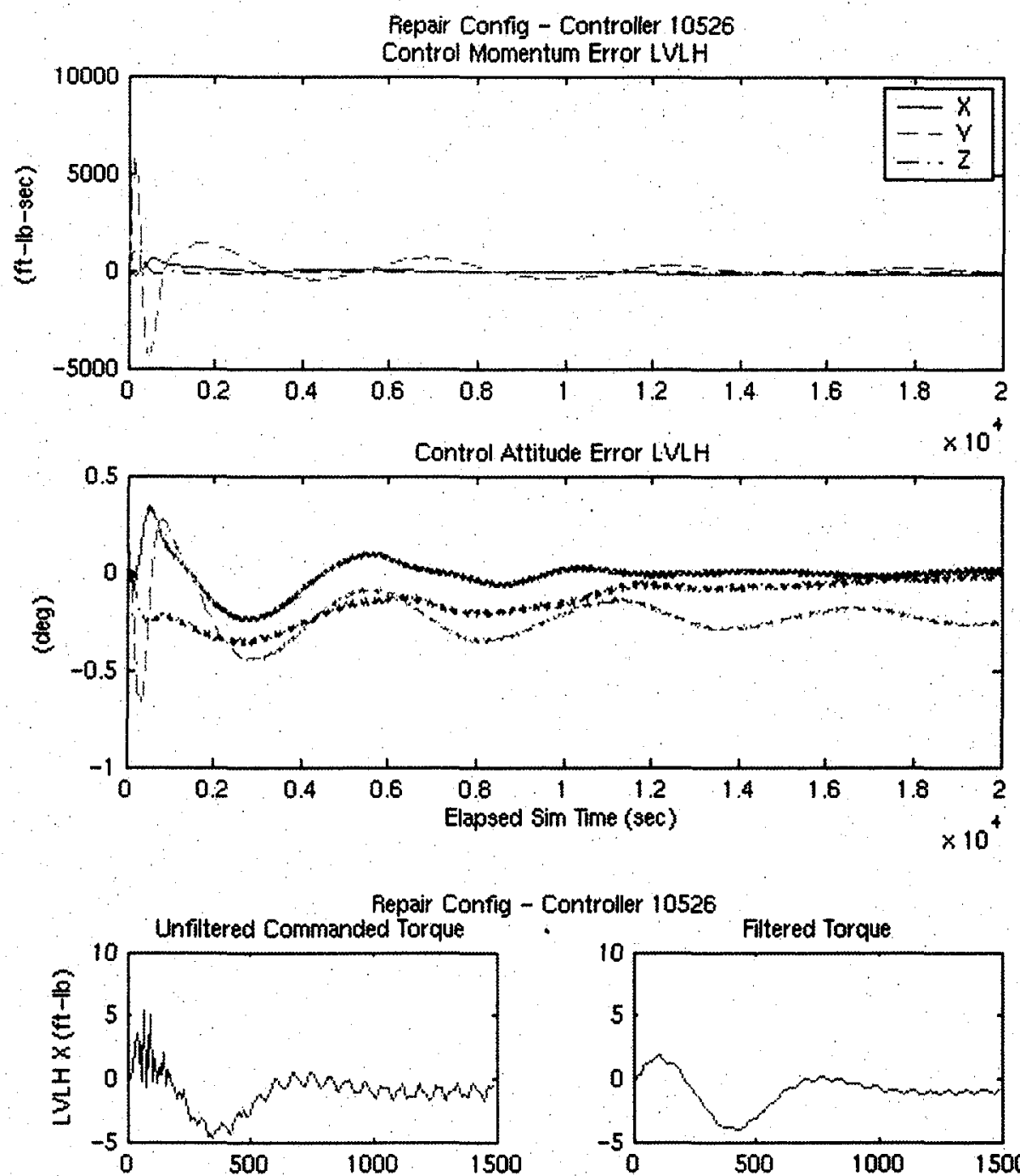

Filtered Torque
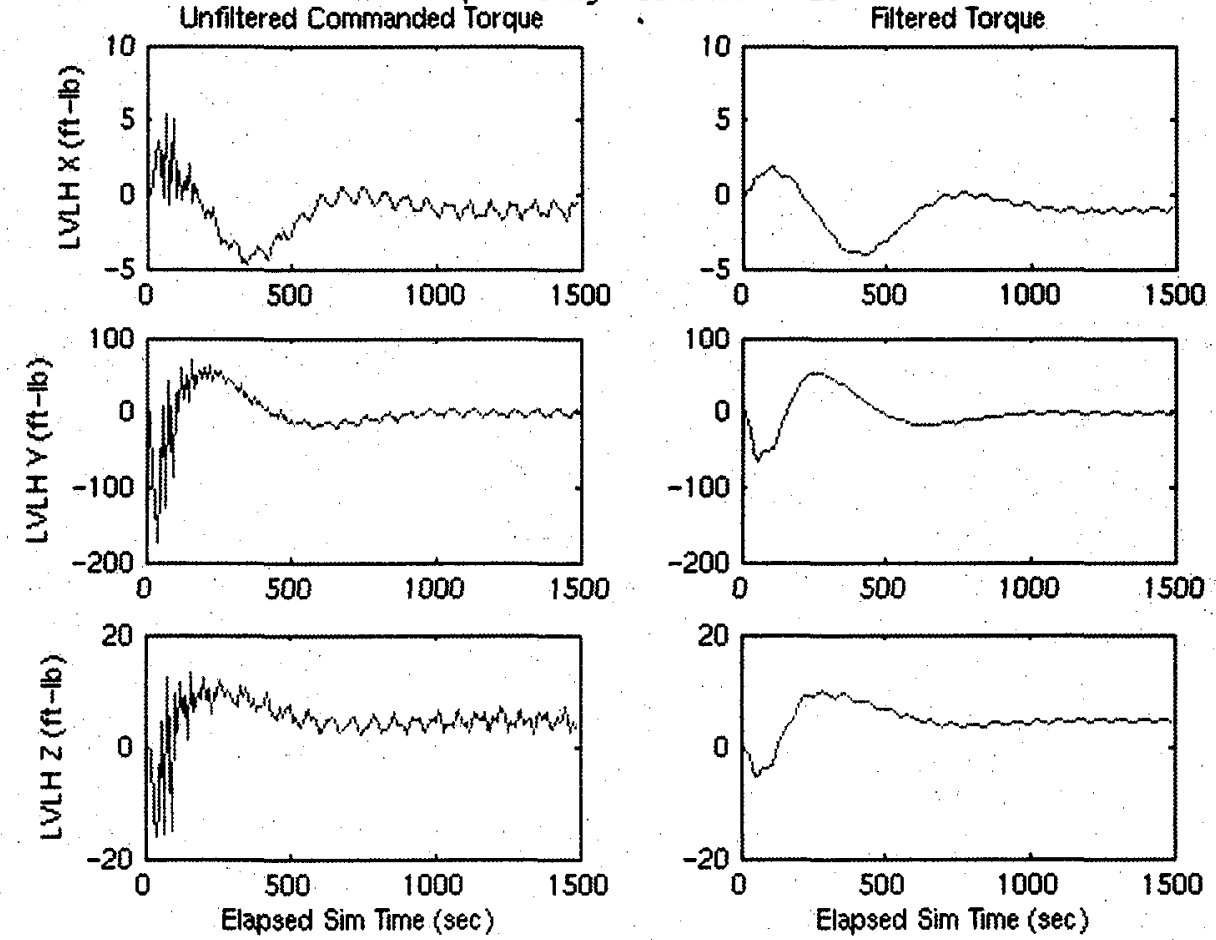


\section{Conclusion}

The work captured in this paper shows that a non-propulsive attitude control capability can be delivered by the ISS U.S. GN\&C system for key ORM and Repair operations. Additional designs were requested later with more momentum margin to support 2 CMG operations, and this work is not detailed in this paper. Future work is to develop a tool that solves for the rigid body state feedback gains and the flex filter parameters simultaneously. Additional future work will be in better linear model parameters for the SRMS flexibility dynamics.

\section{Acknowledgments}

Author Thanks Andy Barth and Karl Sebelius for their analysis of the designs with the SOMBAT tool.

Author Thanks Wayne Warren for his development of modeling mode shape amplitude uncertainty and modal frequency uncertainty in a LFT framework.

\section{References}

${ }^{1}$ Harduvel, J, "Continuous momentum management of earth-oriented spacecraft," , Journal of Guidance, Control, and Dynamics, Vol. 15, No. 6, 1992, pp. 1417-1426.

${ }^{2}$ Boyd, S., El Ghaoui, L., Feron, E., and Balakrishnan, V., Linear Matrix Inequalities in System and Control Theory, SIAM, Philadelphia, 1994, Chaps. 7 